\title{
Excited Electron Dynamics Modeling of Warm Dense Matter
}

\author{
Julius T. Su and William A. Goddard III \\ Materials and Process Simulation Center, Caltech, Pasadena, California, 91125, USA
}

(Received 1 August 2007; published 1 November 2007)

\begin{abstract}
We present a model (the electron force field, or eFF) based on a simplified solution to the timedependent Schrödinger equation that with a single approximate potential between nuclei and electrons correctly describes many phases relevant for warm dense hydrogen. Over a temperature range of 0 to $100000 \mathrm{~K}$ and densities up to $1 \mathrm{~g} / \mathrm{cm}^{3}$, we find excellent agreement with experimental, path integral Monte Carlo, and linear mixing equations of state, as well as single-shock Hugoniot curves from shock compression experiments. In principle eFF should be applicable to other warm dense systems as well.
\end{abstract}

DOI: $10.1103 /$ PhysRevLett.99.185003

Warm dense matter appears in giant planetary interiors [1], inertial confinement fusion [2], and shock [3] or laser [4] heated solids that become plasmas. It is a challenging state to describe theoretically, as it lies in between the cold matter described well by ground state quantum mechanics $[5,6]$, and the hot matter described well by classical plasma models [7]. Reactions, dissociations, and ionizations may occur simultaneously, and excited electron effects may be important.

As a prototype system, we consider the case of hydrogen, which at room temperature remains insulating at pressures as high as $342 \mathrm{GPa}$ [8], but at $3000 \mathrm{~K}$ is already conducting at $140 \mathrm{GPa}$ [9]. This enhanced conductivity of warm dense hydrogen may involve the participation of electronically excited mixtures of $\mathrm{H}_{2}$ molecules, $\mathrm{H}$ atoms, and other species along with free protons and electrons [10].

We present a model (the electron force field, or eFF) based on a simplified solution to the time-dependent Schrödinger equation that with a single approximate potential between nuclei and electrons correctly describes many phases relevant for warm dense hydrogen. eFF computes the energy of a collection of point charge nuclei and single Gaussian function electrons [11] $(\psi=\exp [(\mathbf{r}-$ $\left.\left.\mathbf{x}_{\mathbf{i}}\right)^{2} / s_{i}^{2}\right]$ ) as a function of the nuclear and electron positions plus the electron sizes. It approximates the total energy as the sum of electron kinetic energies, electrostatic potential energies, and Pauli exclusion interactions.

We first assume that electrons move independently of each other, i.e., the wave function is a Hartree product. Then the kinetic energy and electrostatic potential energies of electrons have well-known analytic forms (e.g., $E_{\mathrm{ke}}=3 /\left(2 s^{2}\right), E_{\mathrm{nuc}(i) \cdot \operatorname{elec}(j)}=-Z_{i} R_{i j}^{-1} \operatorname{Erf}\left(\sqrt{2} R_{i j} / s_{j}\right)$, and $\left.E_{\text {elec }(i) \cdot \operatorname{elec}(j)}=r_{i j}^{-1} \operatorname{Erf}\left(\sqrt{2} r_{i j} / \sqrt{s_{i}^{2}+s_{j}^{2}}\right)\right)$. Small electrons have higher kinetic energy, consistent with the Heisenberg uncertainty principle. The finite size of the hydrogen atom, for example, results from the balance between the kinetic energy of the electron and the electrostatic attraction of the electron to the proton.
PACS numbers: 52.27.Gr, 52.25.Kn, 52.35.Tc, 52.65.Yy

However, as electrons are fermions, we make a correction to the independent electron assumption, and estimate a Pauli exclusion energy as a pairwise sum between electrons:

$$
E_{\text {Pauli }}=\sum_{\sigma_{i}=\sigma_{j}} E(\uparrow \uparrow)_{i j}+\sum_{\sigma_{i} \neq \sigma_{j}} E(\uparrow \downarrow)_{i j},
$$

with $\sigma$ denoting the spin of the electrons, and

$$
\begin{aligned}
E(\uparrow \uparrow)_{i j} & =\left(\frac{S_{i j}^{2}}{1-S_{i j}^{2}}+(1-\rho) \frac{S_{i j}^{2}}{1+S_{i j}^{2}}\right) \Delta T_{i j}, \\
E(\uparrow \downarrow)_{i j} & =\frac{\rho S_{i j}^{2}}{1+S_{i j}^{2}} \Delta T_{i j},
\end{aligned}
$$

where $\Delta T$ is a measure of the kinetic energy change upon antisymmetrization, and $S$ is the overlap between two Gaussian functions:

$$
\begin{aligned}
\Delta T_{i j} & =\frac{3}{2}\left(\frac{1}{\bar{s}_{1}^{2}}+\frac{1}{\bar{s}_{2}^{2}}\right)-\frac{2\left(3\left(\bar{s}_{1}^{2}+\bar{s}_{2}^{2}\right)-2 \bar{x}_{12}^{2}\right)}{\left(\bar{s}_{1}^{2}+\bar{s}_{2}^{2}\right)^{2}}, \\
S_{i j} & =\left(\frac{2}{\bar{s}_{i} / \bar{s}_{j}+\bar{s}_{j} / \bar{s}_{i}}\right)^{3 / 2} \exp \left(-\bar{x}_{i j}^{2} /\left(\bar{s}_{i}^{2}+\bar{s}_{j}^{2}\right)\right),
\end{aligned}
$$

where $\rho=-0.2, \bar{x}_{i j}=x_{i j} \times 1.125$, and $\bar{s}_{i}=s_{i} \times 0.9$. This expression was derived by considering the difference between the antisymmetric and symmetric combinations of valence bond states, and assuming that kinetic energy differences predominate; analogous approximations have been reported elsewhere [12-14].

To compute the ground state, we minimize the energy of the system, optimizing the nuclear and electron positions and the electron sizes simultaneously [15]. We chose the Pauli potential to obtain stable bonds between elements from $Z=1-6$. The specific form selected for the Pauli potential distinguishes eFF from a method such as wave packet molecular dynamics (WPMD [12]), which does not produce stable molecules with elements $Z>1$ and does not describe the equation of state (EOS) of dense hydrogen accurately. We optimized the three universal parameters of the Pauli potential to reproduce the bond lengths of small molecules such as $\mathrm{CH}_{4}[1.143$ (1.094) $\AA$, exact values in 
parentheses $], \quad \mathrm{C}_{2} \mathrm{H}_{6} \quad\left[d_{\mathrm{CC}}=1.501(1.536) \AA, \quad d_{\mathrm{CH}}=\right.$ 1.173(1.091) $\mathrm{A}]$, $\mathrm{LiH}[1.689(1.596) \AA]$, and $\mathrm{B}_{2} \mathrm{H}_{6}\left[d_{\mathrm{BB}}=\right.$ 1.744(1.757) $\AA, \quad d_{\mathrm{BH} 1}=1.347(1.331) \AA, \quad d_{\mathrm{BH} 2}=$ $1.243(1.207) \AA$. No further optimization of parameters was used in simulating warm dense hydrogen.

Applied to the ground state, eFF leads to reasonable covalent bonds in $\mathrm{H}_{2} \quad\left[d_{\text {bond }}=0.780(0.741) \AA\right.$, $E_{\text {bond }}=67.2(104.2) \mathrm{kcal} / \mathrm{mol}$, exact values in parentheses] and metallic bonding in lithium metal $\left[a_{\mathrm{fcc}}=\right.$ 4.401(4.420) $\left.\AA, E_{\text {atomize }}=60.3(37.7) \mathrm{kcal} / \mathrm{mol}\right]$. For $\mathrm{H}_{2}$ at equilibrium the covalent bond is a bond-centered Gaussian, while in face-centered cubic lithium the metallic bonds are Gaussians located in octahedral interstitial locations coordinating six Li ions. The ability of the Gaussian functions to move and change size provides them with valuable variational flexibility [11].

To simulate materials at finite temperatures, we propagate semiclassical electron dynamics $[12,13,16,17]$, allowing the positions of the nuclei and electrons, as well as the sizes of the electrons, to change with time. Allowing electrons to grow and shrink leads to important effectsfor example, warm hydrogen atoms have larger electrons than cold hydrogen atoms, which causes increased Pauli repulsion between atoms.

In the dynamics simulations, we represent electrons as wave packets:

$$
\Psi \propto \exp \left[-\left(\frac{1}{s^{2}}-\frac{2 p_{s}}{s} i\right)(\mathbf{r}-\mathbf{x})^{2}\right] \exp \left[i \mathbf{p}_{\mathbf{x}} \cdot \mathbf{r}\right] .
$$

Substituting the above expression into the time-dependent Schrödinger equation and assuming a locally harmonic potential produces the semiclassical equations of motion:

$$
\begin{array}{rlrl}
\dot{p}_{x}=-\nabla_{x} V, & \mathbf{p} & =m_{\text {elec }} \dot{\mathbf{x}}, \\
\dot{p}_{s}=-\partial V / \partial s, & p_{s}=\left(3 m_{\text {elec }} / 4\right) \dot{s} .
\end{array}
$$

Thus the average position of a wave packet obeys classical dynamics (Ehrenfests' theorem) with the addition that the size of the wave packet obeys classical dynamics as well [13]. With this method, the size and position of each electron evolves independently, and we can obtain nonadiabatic electron dynamics if desired.

We extract thermodynamic parameters from the simulations using classical virial expressions, e.g., $\frac{3}{2} k_{B} T=\frac{1}{N_{\text {nuc }}} \times$ $\left\langle\sum_{i} \frac{1}{2} m_{i} v_{i}^{2}\right\rangle$ and $P V=N_{\text {nuc }} k_{B} T+\frac{1}{3}\left\langle\sum_{i} x_{i} \frac{\partial E}{\partial x_{i}}\right\rangle$, where we sum over all nuclear and electronic degrees of freedom and take $m_{\text {elec }}=m_{\text {nuc }}$. In order to compute quantities dependent solely on the electronic degrees of freedom, such as plasma oscillation frequencies or electrical conductivity, a more rigorous separation of nuclear and electronic time scales is likely required.

Figure 1 shows the equation of state of solid deuterium at $300 \mathrm{~K}$ computed using semiclassical dynamics with eFF. We indicate density with the Wigner-Seitz radius $r_{s}$, where each atom occupies a volume $\frac{4}{3} \pi r_{s}^{3}$. Pressure versus density data has been measured up to $120 \mathrm{GPa}\left(r_{s}=\right.$

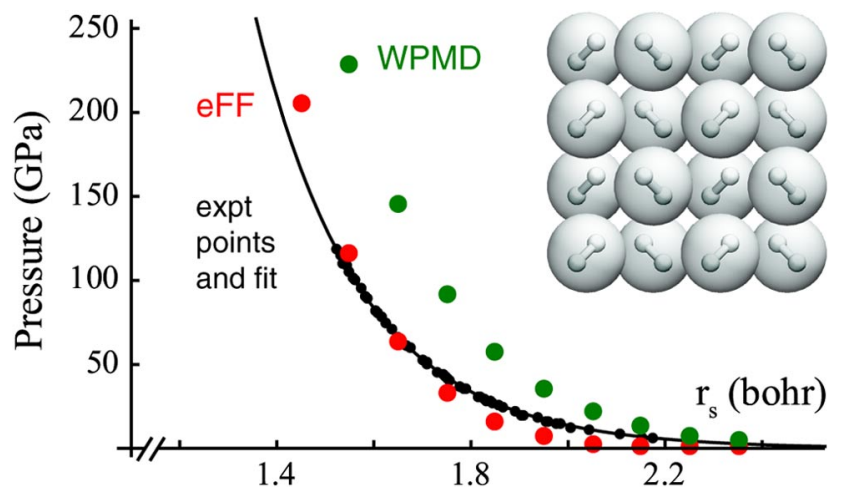

FIG. 1 (color online). Equation of state for solid deuterium at $300 \mathrm{~K}$ and varying density. We show here that the pressures from eFF dynamics are in good agreement with diamond anvil experiments. Here constant number/volume/energy (NVE) dynamics were performed in a cubic periodic box with $64 \mathrm{H}$ atoms. Electrostatics were computed using Ewald summation, and $E_{\text {Pauli }}$ was computed using the minimum image convention. Thermodynamic parameters were averaged over $1 \mathrm{ps}$ following a $200 \mathrm{fs}$ equilibration period, with the time step set to $\min (0.02$, $1 / \sqrt{T})$ fs. Masses were set to $m_{\text {nuc }}=m_{\text {elec }}=1.008 \mathrm{amu}$; reducing the electron mass to $m_{\text {nuc }}=0.5 \mathrm{amu}$ and increasing the nuclear mass by the same amount had a negligible effect on the equations of state.

$1.52 \mathrm{bohr}$ ) at $300 \mathrm{~K}$ using diamond anvil apparatuses [18]. The equation of state from eFF is in good agreement with the results obtained over the pressure range of the experiment.

We now consider the behavior of hydrogen at higher temperatures. At $1 \mathrm{~atm}, \mathrm{H}_{2}$ dissociates into atoms at $3960 \mathrm{~K}$ and the atoms ionize at $15870 \mathrm{~K}$ (from the Saha equation [19], which assumes an ideal gas). In the ideal gas limit, the dissociation and ionization temperatures increase with density, remaining separate events. To study the nonideal behavior of warm dense hydrogen, Saumon and Chabrier constructed a linear mixing EOS [20] taking into account contributions from molecular, atomic, ionized, and metallic phases. Their model predicts that the ionization temperature lowers to coincide with the dissociation temperature in a plasma phase transition at $r_{s}=$ 2 bohr and $T=15300 \mathrm{~K}$.

Figure 2 shows the pair distribution predicted from eFF as deuterium is heated from 9100 to $30720 \mathrm{~K}$ at $r_{s}=$ 2 bohr. We find that deuterium gradually dissociates from fluid molecules to fluid atoms over this temperature range. Fluid molecules are characterized by strong peaks in the proton-proton pair distribution at the $\mathrm{D}_{2}$ bond distance and first solvation shell ( 0.74 and $2.3 \AA$ ), while fluid atoms are characterized by a pair distribution that rises gradually as $r$ increases from 0.5 to $1.5 \AA$ and then is a constant for longer distances. The eFF midpoint of dissociation is at $T=16500 \mathrm{~K}$ (where $r_{\min } / r_{\max }=0.5$ ), comparable to the dissociation temperature $(15300 \mathrm{~K})$ predicted by Saumon and Chabrier. 


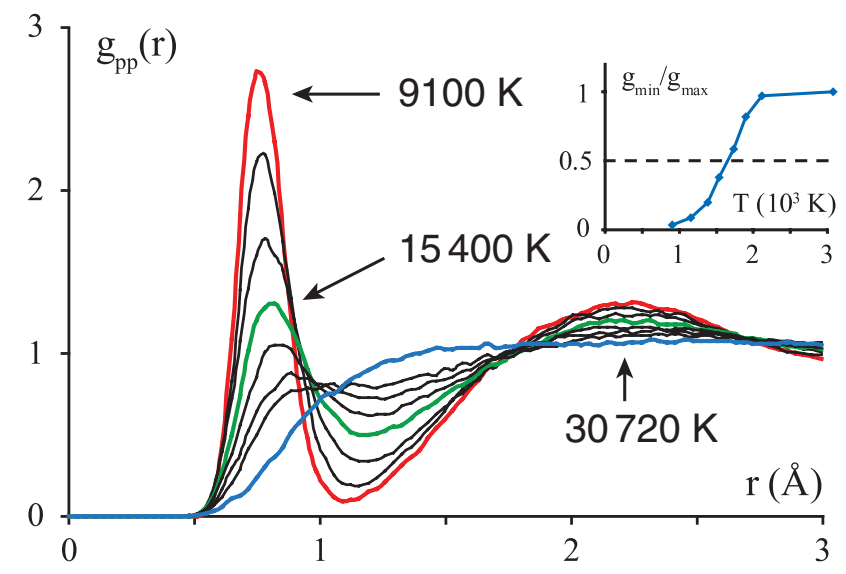

FIG. 2 (color online). Proton-proton pair distribution function for liquid $\mathrm{D}_{2}$ for $r_{s}=2$ bohr and varying temperature. We show here that eFF predicts a gradual dissociation of molecules into separated atoms as the temperature increases from $T=$ $10000 \mathrm{~K}$ to $20000 \mathrm{~K}$. The inset shows the ratio of the amplitude at the first minimum $\left(g_{\min }\right)$ to the amplitude at the first maximum $\left(g_{\max }\right)$, indicating that the midpoint is at $16500 \mathrm{~K}$. The dynamics parameters were the same as in Fig. 1.

From eFF dynamics, the average electron size increases with temperature, from $1.74 \mathrm{bohr}$ at $4200 \mathrm{~K}$ to $2.32 \mathrm{bohr}$ at $33000 \mathrm{~K}$. At temperatures exceeding $\sim 50000 \mathrm{~K}$, some electrons begin to expand past the dimensions of the periodic box, which we consider to represent ionization [21]. This suggests that at $r_{s}=2 \mathrm{bohr}$, dissociation and ionization occur in separate temperature ranges, $16500 \mathrm{~K}$ and $50000 \mathrm{~K}$, respectively.

Figure 3 shows the pressure versus temperature of $\mathrm{D}_{2}$ at $r_{s}=2$ bohr. Since no experimental data are available, we compare eFF to equations of state from the most accurate $a b$ initio theories. Path integral Monte Carlo (PIMC) is in principle exact but in practice the accuracy is limited by the step size, which is inversely proportional to temperature. PIMC predicts that the midpoint of molecular dissociation occurs at $10000 \mathrm{~K}$; however it also predicts that $d P / d T<$ 0 at this temperature, which initially [22] was taken as evidence of a first-order phase transition. But later [6] this result was concluded to be an unphysical artifact of the simulation. Thus currently PIMC can capture accurately only the thermodynamics of higher temperature dissociated states [6]. At lower temperatures $(<10000 \mathrm{~K})$, we compare our results against the Saumon-Chabrier EOS, which used parameters fitted to describe the molecular phase.

Thus eFF provides a unified and continuous description of both the molecular and atomic regimes, including the ionized regime above $50000 \mathrm{~K}$. It matches the pressures from the Saumon-Chabrier EOS well at low temperatures, and it matches the slope of PIMC computations [23] at higher temperatures. At higher temperatures the eFF EOS inflects slightly upward while the PIMC and chemical models both inflect downward over the dissociation regime. We believe that the upward inflection is physically

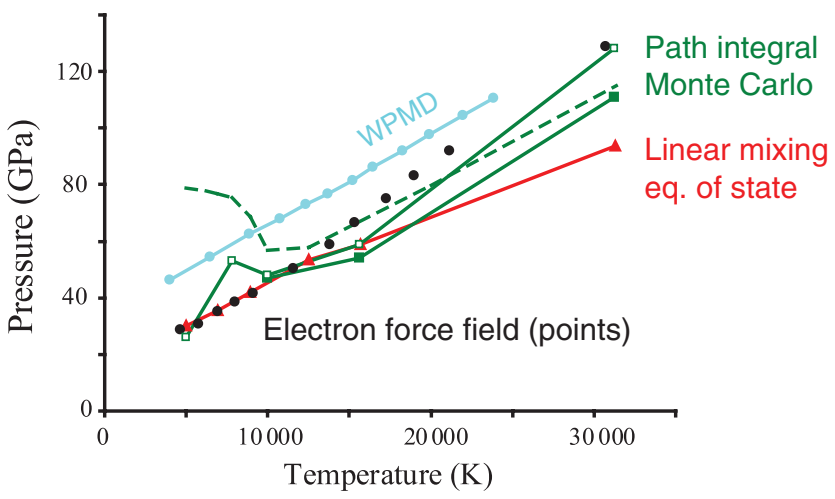

FIG. 3 (color). Equation of state for liquid deuterium at $r_{s}=$ 2 bohr with varying temperature. We show here that eFF is applicable and consistent with the best theory over the entire temperature range shown. The eFF results (black round points) are in the range with the best QM (PIMC, green solid and dashed lines) above $10000 \mathrm{~K}$ (where PIMC is accurate), and eFF agrees well with the Saumon-Chabrier EOS [20] below $10000 \mathrm{~K}$ (where it is expected to be accurate). The PIMC results shown here are: (a) calculations using free-particle nodes (dashed line [22]), and (b) calculations using antisymmetrized local Gaussian nodes (solid line, open squares [29] and closed squares [23]). Above $T>50000 \mathrm{~K}$ the eFF uses a harmonic restraint to limit the electron size $\left(E=\frac{1}{2} k_{s} s^{2}\right.$ for $\left.s>L_{\text {box }} / 2\right)$; however, the equation of state is insensitive to the particular value of $k_{s}$, as long as it keeps the electrons bound. The eFF pressures from $k_{s}=1$ and from 10 hartrees $/$ bohr $^{2}$ are within $1 \%$ of each other over $T=6000$ to $600000 \mathrm{~K}$.

reasonable, since the doubling in the number of free particles resulting from dissociation should increase the pressure. While eFF describes the dissociation of molecules into atoms as the temperature increases, it does not show the discontinuous first derivative in the equation of state that would characterize a first-order plasma phase transition.

The good agreement of eFF with PIMC persists to $\sim 100000 \mathrm{~K}$, above which point the eFF EOS slope becomes too small, with the pressure becoming $20 \%$ too low by $120000 \mathrm{~K}$. Thus the simple eFF, with no adjustable parameters, provides a consistent description of hydrogen over the 0 to $100000 \mathrm{~K}$ temperature range.

Figure 4 shows the eFF computed pressure-density relation (Hugoniot curve) of shocked liquid deuterium, where deuterium is heated from $20 \mathrm{~K}$ to tens of thousands of degrees within tens of nanoseconds. The single-shock Hugoniot curve for liquid deuterium is by now well established, with experimental data from diverse shock sources such as gas guns [24], exploding wires ( $Z$ machine [25]), and imploding hemispheres (convergent geometry [26]), as well as theoretical results from PIMC in impressive accord with each other. These data support a curve that sharply inflects upward at a maximum compressibility of $\rho / \rho_{0} \sim$ 4 , consistent with the limiting compressibility of other diatomics such as $\mathrm{CO}$ and $\mathrm{N}_{2}$ [27]. The redundant experiments were spurred by controversy over an anomalously 


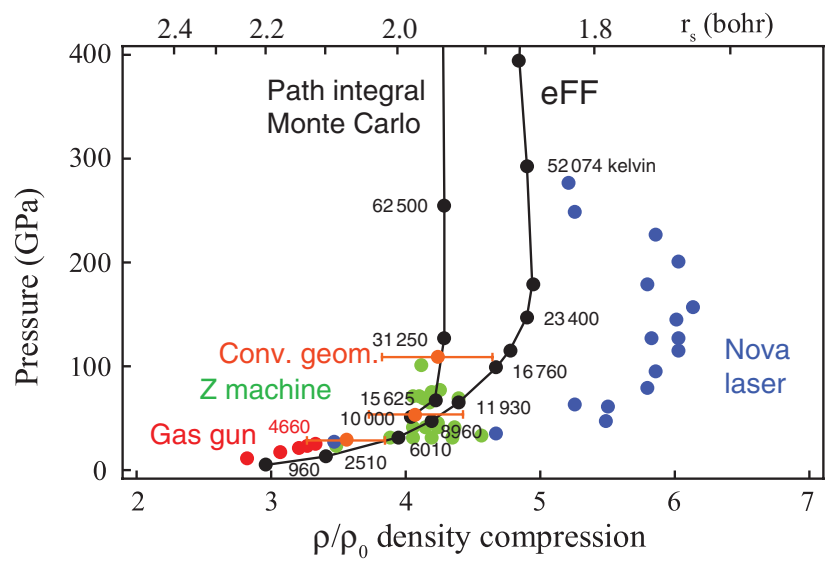

FIG. 4 (color). Shock Hugoniot curve for liquid $\mathrm{D}_{2}$. We show here that eFF agrees well with most experiments: gas gun (red dots), $Z$ machine (green dots), and convergence geometry (orange). The validity of the Nova laser data (blue dots) has been questioned [27]. The PIMC results agree with eFF up to a compression of 4.2 , but leads to a lower limiting compression than eFF. To compute the Hugoniot curve, we perform NVE simulations of $\mathrm{D}_{2}$, interpolating to temperatures such that the internal energy, volume, and pressure satisfy $U-U_{0}+\frac{1}{2}(V-$ $\left.V_{0}\right)\left(P+P_{0}\right)=0$. As a starting point, we compute a box of liquid hydrogen with $r_{s}=3.16 \mathrm{bohr}\left(\rho_{0}=0.171 \mathrm{~g} / \mathrm{cm}^{3}\right), T=$ $19.6 \mathrm{~K}$; we find $U_{0}=-0.477043$ hartrees/atom and $P=0$. We note that the eFF Hugoniot curve connects to an eFF low temperature starting point, while the PIMC Hugoniot curve connects to a $U_{0}$ from a separate calculation [30].

large compressibility $\rho / \rho_{0}=6 \pm 1.8$ observed using laser ablation as a shock source (Nova laser [28]). The latest convergent geometry experiments have the smallest error bars, and definitively support a maximum compressibility of $4 \pm 0.6$.

The eFF Hugoniot passes through the gas gun and $Z$ machine data points, and shows a stiff upward bend, but predicts a maximum compressibility of 4.8 , which lies slightly above the limits of 3.4 to 4.6 from the convergence geometry experiments. Below $50 \mathrm{GPa}$, the eFF Hugoniot curve leads pressures and temperatures that are slightly too low (eFF $P=13 \mathrm{GPa}, T=2510 \mathrm{~K}$ at $r_{s}=2.00$ bohr versus gas gun $P=25 \mathrm{GPa}, T=4650 \mathrm{~K}$ at $r_{s}=2.10 \mathrm{bohr}$ ), but the upward slope matches experiment. Above $120 \mathrm{GPa}$, the eFF Hugoniot curve bends upward to form a stiff curve with a minimum and maximum $\rho / \rho_{0}$ of 4.8 and 5.0. In this pressure range, the PIMC Hugoniot curve is shaped like a backward C, with a maximum $\rho / \rho_{0}$ of 4.3 which decreases to 4.1 at $P=5608 \mathrm{GPa}\left(T=10^{6} \mathrm{~K}\right)$. Hence the eFF Hugoniot curve has the correct overall shape, but the maximum compressibility is slightly too high.

The encouraging results obtained with warm dense hydrogen, as well as the correct ground state geometries obtained for molecules with elements from $Z=1-6$, suggest that eFF could be a valuable and general method for studying the excited electron dynamics of systems with diverse combinations of elements and bonding. The eFF potential contains terms no more complex than those used in traditional force fields involving only nuclei. Thus it should be practical to use eFF to study large scale excited systems beyond the reach of current quantum mechanics. We anticipate further developments will be required for applications to atoms beyond carbon.

We thank Professor William J. Nellis for useful comments. This work was supported by the U.S. DOE (ASC) and DARPA-ONR (PROM).

[1] H. M. van Horn, Science 252, 384 (1991).

[2] M. K. Matzen et al., Phys. Plasmas 12, 055503 (2005).

[3] R. Cauble et al., Contrib. Plasma Phys. 41, 239 (2001).

[4] R. W. Lee et al., J. Opt. Soc. Am. B 20, 770 (2003).

[5] L. Collins et al., Phys. Rev. E 52, 6202 (1995).

[6] K. T. Delaney, C. Pierleoni, and D. M. Ceperley, Phys. Rev. Lett. 97, 235702 (2006).

[7] J. M. Dawson, Rev. Mod. Phys. 55, 403 (1983).

[8] C. Narayana et al., Nature (London) 393, 46 (1998).

[9] S. T. Weir, A.C. Mitchell, and W. J. Nellis, Phys. Rev. Lett. 76, 1860 (1996).

[10] W. J. Nellis, S. T. Weir, and A. C. Mitchell, Phys. Rev. B 59, 3434 (1999).

[11] A. A. Frost, J. Chem. Phys. 47, 3707 (1967).

[12] D. Klakow, C. Toepffer, and P.-G. Reinhard, J. Chem. Phys. 101, 10766 (1994).

[13] M. Knaup, P.-G. Reinhard, C. Toepffer, and G. Zwicknagel, J. Phys. A 36, 6165 (2003).

[14] D. H. Boal and J. N. Glosi, Phys. Rev. C 38, 1870 (1988); L. Wilets, E. M. Henley, M. Kraft, and A. D. Mackellar, Nucl. Phys. A282, 341 (1977); C. Dorso, S. Duarte, and J. Randrup, Phys. Lett. B 188, 287 (1987).

[15] See EPAPS Document No. E-PRLTAO-99-085743 for coordinates and other supporting information. For more information on EPAPS, see http://www.aip.org/pubservs/ epaps.html.

[16] E. J. Heller, J. Chem. Phys. 62, 1544 (1975).

[17] H. Feldmeier and Schnack, Rev. Mod. Phys. 72, 655 (2000).

[18] P. Loubeyre et al., Nature (London) 383, 702 (1996).

[19] M. N. Saha, Philos. Mag. 40, 72 (1920).

[20] D. Saumon and G. Chabrier, Phys. Rev. Lett. 62, 2397 (1989).

[21] More models: K. A. H. van Leeuwen et al., Phys. Rev. Lett. 55, 2231 (1985); D. A. Wasson and S.E. Koonin, Phys. Rev. A 39, 5676 (1989).

[22] W. R. Margo, D. M. Ceperley, C. Pierleoni, and B. Bernu, Phys. Rev. Lett. 76, 1240 (1996).

[23] B. Militzer and D. M. Ceperley, Phys. Rev. Lett. 85, 1890 (2000).

[24] W. J. Nellis et al., J. Chem. Phys. 79, 1480 (1983).

[25] M. D. Knudson et al., Phys. Rev. Lett. 90, 035505 (2003).

[26] G. V. Boriskov et al., Phys. Rev. B 71, 092104 (2005).

[27] W. J. Nellis, Phys. Rev. Lett. 89, 165502 (2002).

[28] G. W. Collins et al., Science 281, 1178 (1998).

[29] B. Militzer, Ph.D. thesis, University of Illinois at UC 2002.

[30] W. Kolos and L. Wolniewicz, J. Chem. Phys. 41, 3674 (1964). 